\title{
ON THE DISTRIBUTION OF ALEXANDER POLYNOMIALS OF ALTERNATING KNOTS AND LINKS
}

\author{
SHIN'ICHI KINOSHITA
}

\begin{abstract}
In this paper we will be concerned with the distribution of the reduced Alexander polynomials of alternating knots and links. Our theorems are quite different in style from those of R. H. Crowell, K. Murasugi and R. Riley, which intend to characterize the reduced Alexander polynomials of alternating knots and links.
\end{abstract}

1. A link $l$ is a finite collection of mutually disjoint oriented polygonal simple closed curves in the 3-space $R^{3}$. Two links $l$ and $l_{1}$ are called topologically equivalent, if there is an autohomeomorphism of $R^{3}$, which carries $l$ onto $l_{1}$. In this paper we will not clearly distinguish a link and its equivalence class.

For each link $l$ we associate a polynomial $\Delta_{l}(t)$, introduced by $\mathrm{J}$. W. Alexander [1]. The polynomial $\Delta_{l}(t)$ is actually an element of the group-ring $J H$ of the infinite cyclic group $H$ generated by $t$ over integers. The polynomial $\Delta_{l}(t)$ is determined up to $\pm t^{\lambda}$, which is a unit in $J H$. (See R. H. Crowell and R. H. Fox [4].)

In the case of a knot $k$, i.e., a link with one component, the polynomial $\Delta_{k}(t)$ is called the Alexander polynomial of $k$ and satisfies the following conditions:

(1) $\Delta_{k}(1)= \pm 1$, and

(2) $\Delta_{k}(t)$ satisfies symmetry and has even reduced degree.

Here symmetry of $\Delta_{k}(t)$ means $\Delta_{k}(t)=t^{\lambda} \Delta_{k}\left(t^{-1}\right)$ for an integer $\lambda$ and the reduced degree of $\Delta_{k}(t)$ is the difference between the highest degree and the lowest degree of the terms with nonzero coefficients. Condition (1) is proved by Alexander [1] and condition (2) is proved by $H$. Seifert [12]. Further, by Seifert [12], these two conditions are sufficient for a polynomial $\Delta(t)$ to be the Alexander polynomial of a knot.

In the case of a link $l$ with a number of components greater than one, the polynomial $\Delta_{l}(t)$ is called the reduced Alexander polynomial of $l$ and satisfies the following conditions:

(3) $\Delta_{l}(t)=(t-1)^{\mu-1} \nabla_{l}(t)$, where $\mu$ is the number of components of $l$, and

(4) $\nabla_{l}(t)$ satisfies symmetry and has even reduced degree.

Condition (3) can easily be seen (see G. Torres [13]) and condition (4) is proved by Torres [13] and F. Hosokawa [6]. By Hosokawa [6], these two conditions are sufficient for a polynomial $\Delta(t)$ to be the reduced Alexander polynomial of a link.

Received by the editors May 4, 1978. The paper was presented at the Regional Conference on Three-Manifold Topology, Virginia Polytechnic Institute and State University, October 8-13, 1977.

AMS (MOS) subject classifications (1970). Primary 55A25.

Key words and phrases. Alternating knots and links, Alexander polynomials. 
In this paper we will call the Alexander polynomial of a knot the reduced Alexander polynomial, also.

2. Let $l$ be a link and $L$ its regular projection on a plane. For each segment of $L$ which passes through a double point of $L$, we associate the assignment of overpass or underpass at that point. A link $l$ is called alternating, if an appropriate regular projection of $l$ has the property such that when a point moves on each of the components of $l$, its projection passes through the double points in such a way that the assignment of overpass and underpass appears alternately.

The collection of all alternating links forms a proper subset of the set of all links. The reduced Alexander polynomial of an alternating link satisfies some additional requirements so that not all reduced Alexander polynomials of links are those of alternating links. These requirements are investigated by $\mathbf{R}$. H. Crowell ([2], [3]), K. Murasugi ([8], [9], [10]) and R. Riley [11], but a complete characterization of the reduced Alexander polynomials of alternating links is still unknown.

Suppose that a link $l$ is given and let $L$ be its regular projection on a plane. Then one can choose some double points of $L$ in such a way that if we interchange the assignment of overpass and underpass at each of these double points, the assignment of overpass and underpass appears alternately. Hence, the projection $L$ with these new assignments is a regular projection of an alternating link.

3. Let $L_{1}$ be a regular projection of a link $l_{1}$ on a plane. Choose a double point of $L_{1}$ and apply the operation described as in Figure 1 or Figure 2 at this double point. Then $L_{1}$ is changed to $L_{2}$, which is a regular projection of a link $l_{2}$.

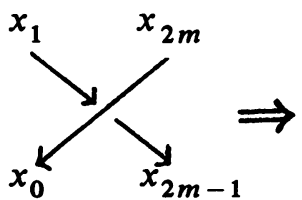

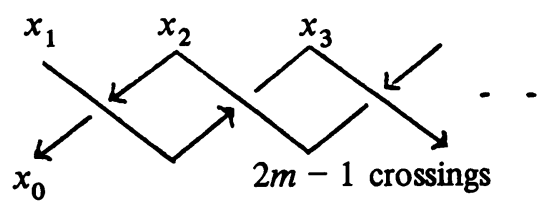

FIGURE 1
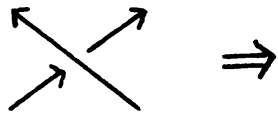

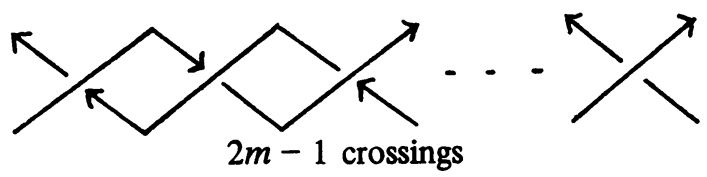

FIGURE 2

THEOREM 1. For appropriate choices of $\Delta_{l_{1}}(t)$ and $\Delta_{l_{2}}(t)$ we have

$$
\Delta_{l_{1}}(t) \equiv \Delta_{l_{2}}(t) \bmod m(1-t) .
$$

Proof. We will only prove the case corresponding to the operation described as in Figure 1. The other case can be proved similarly.

We will compare the fundamental groups and their reduced Alexander matrices of $l_{1}$ and $l_{2}$. We will use the Wirtinger's presentation. From the left-hand side of Figure 1 we have relations

$$
x_{2 m-1}=x_{0} x_{1} x_{0}^{-1} \text { and } x_{2 m}=x_{0}
$$


which induce the entries of the reduced Alexander matrix of $l_{1}$ as follows:

$$
\begin{aligned}
& \begin{array}{llll}
x_{0} & x_{1} & x_{2 m-1} & x_{2 m}
\end{array} \\
& {\left[\begin{array}{cccc}
1-t & t & -1 & 0 \\
1 & 0 & 0 & -1
\end{array}\right] \text {. }}
\end{aligned}
$$

From the right-hand side of Figure 1 we have relations

$$
\begin{aligned}
x_{2 m-1} & =\left(x_{1} x_{0}^{-1}\right)^{m-1} x_{1}\left(x_{1} x_{0}^{-1}\right)^{1-m}, \\
x_{2 m} & =\left(x_{1} x_{0}^{-1}\right)^{m-1} x_{1} x_{0} x_{1}^{-1}\left(x_{1} x_{0}^{-1}\right)^{1-m} .
\end{aligned}
$$

These two relations induce the entries of the reduced Alexander matrix of $l_{2}$ as follows:

$$
\left[\begin{array}{cccc}
x_{0} & x_{1} & x_{2 m-1} & x_{2 m} \\
-(m-1)(1-t) & m(1-t)+t & -1 & 0 \\
-m(1-t)+1 & m(1-t) & 0 & -1
\end{array}\right] .
$$

Hence we have Matrix (1) = Matrix (2) $\bmod m(1-t)$. Since the other double points of $L_{1}$ and $L_{2}$ remain unchanged, we have $\Delta_{l_{1}}(t) \equiv \Delta_{l_{2}}(t) \bmod m(1-t)$. Hence the proof of Theorem 1 is complete.

COROllary. Under the same assumption as above we have $\left|\Delta_{l_{1}}(-1)\right| \equiv\left|\Delta_{l_{2}}(-1)\right|$ $\bmod 2 m$.

4. Let $L_{1}$ be a regular projection of a link $l_{1}$ on a plane. Choose a double point of $L_{1}$ and apply the operation described as in Figure 3 or Figure 4 at this double point. Then $L_{1}$ is changed to $L_{2}$, which is a regular projection of a link $l_{2}$. Let $\sigma_{m}(t)=1+t+t^{2}+\cdots+t^{m-1}$.
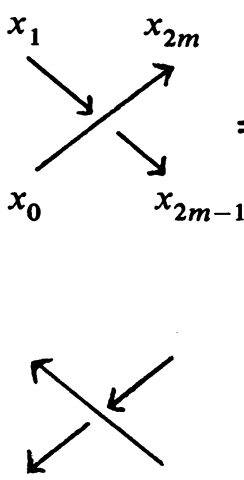

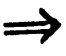

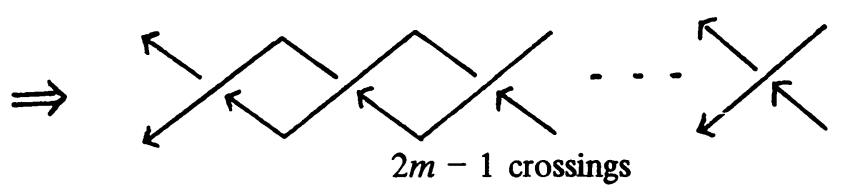

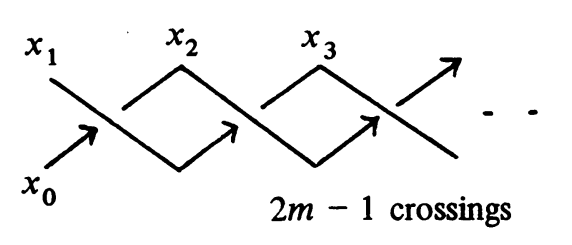

FIgURE 3

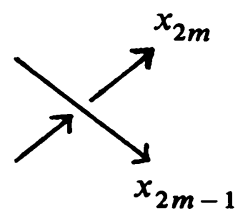

$x_{2 m-1}$

Figure 4

THEOREM 2. For an appropriate choice of $\Delta_{l_{1}}(t)$ and $\Delta_{l_{2}}(t)$ we have $\Delta_{l_{1}}(t) \equiv \Delta_{l_{2}}(t)$ $\bmod (1-t) \sigma_{m}\left(t^{2}\right)$.

Proof. We will only prove the case corresponding to the operation described as in Figure 3. The other case can be proved similarly. 
As before, we will compare the fundamental groups and their reduced Alexander matrices of $l_{1}$ and $l_{2}$. From the left-hand side of Figure 3 we have relations

$$
x_{2 m-1}=x_{0}^{-1} x_{1} x_{0} \text { and } x_{2 m}=x_{0}
$$

and the corresponding entries of the reduced Alexander matrix

$$
\left.\begin{array}{cccc}
x_{0} & x_{1} & x_{2 m-1} & x_{2 m} \\
1-t^{-1} & t^{-1} & -1 & 0 \\
1 & 0 & 0 & -1
\end{array}\right]
$$

From the right-hand side of Figure 3 we have relations

$$
\begin{aligned}
x_{2 m-1} & =\left(x_{1} x_{0}\right)^{m-1} x_{1}\left(x_{1} x_{0}\right)^{1-m}, \\
x_{2 m} & =\left(x_{1} x_{0}\right)^{m-1} x_{1} x_{0} x_{1}^{-1}\left(x_{1} x_{0}\right)^{1-m},
\end{aligned}
$$

and the corresponding entries of the reduced Alexander matrix

$$
\left[\begin{array}{cccc}
x_{0} & x_{1} & x_{2 m-1} & x_{2 m} \\
1-t^{2 m-1}-(1-t) \sigma_{m}\left(t^{2}\right) & t^{2 m-1}+(1-t) \sigma_{m}\left(t^{2}\right) & -1 & 0 \\
1-(1-t) \sigma_{m}\left(t^{2}\right) & (1-t) \sigma_{m}\left(t^{2}\right) & 0 & -1
\end{array}\right]
$$

Note that we have $1-t^{2 m}=\left(1-t^{2}\right) \sigma_{m}\left(t^{2}\right)$ and hence

$$
t^{2 m-1} \equiv t^{-1} \bmod (1-t) \sigma_{m}\left(t^{2}\right) \text {. }
$$

Therefore we have Matrix (3) $\equiv$ Matrix (4) $\bmod (1-t) \sigma_{m}\left(t^{2}\right)$. Since the other double points of $L_{1}$ and $L_{2}$ remain unchanged, we have

$$
\Delta_{l_{1}}(t) \equiv \Delta_{l_{2}}(t) \bmod (1-t) \sigma_{m}\left(t^{2}\right) .
$$

Thus the proof of Theorem 2 is complete.

Corollary. Under the same assumption as above we have $\left|\Delta_{l_{1}}(-1)\right| \equiv\left|\Delta_{l_{2}}(-1)\right|$ $\bmod 2 m$.

REmark 1. Corollaries of Theorems 1 and 2 can be seen easily by the use of the determinant of knots and links.

Remark 2. In [5], R. H. Fox proved the same kind of theorem as Theorems 1 and 2 above, but in a more general setting. Though there is an error in his proof, the theorem turns out to be correct.

A proof of a theorem in [7] by the author of this paper following the same pattern as Fox is also in error. A counterexample to the theorem in [7] was shown to the author by S. Suzuki.

5.

TheOREM 3. Let $m$ be a positive integer and $f(t)$ an integral polynomial in $t$. Assume that $f(t)$ satisfies conditions (1) and (2) in the case for a knot and conditions (3) and (4) in the case for a link with the number of components greater than 1. Then there exists an alternating link $l$ with the given number of components such that $\Delta_{l}(t) \equiv f(t) \bmod m(1-t)$. 
Proof. By Seifert [12] and Hosokawa [6], there exists a link $l_{1}$ with the given number of components such that $\Delta_{l_{1}}(t)=f(t)$. Let $L_{1}$ be a regular projection of $l_{1}$ on a plane. Choose double points of $L_{1}$ in such a way that if we interchange the overpass and underpass at each of these double points, $L_{1}$ is changed to $L_{2}$, which is a regular projection of an alternating link. Now apply one of the operations described as in Figures 1 and 2 at each of these double points of $L_{1}$. If we choose the appropriate operation at each of these double points, $L_{1}$ is changed to $L$, which is a regular projection of an alternating link $l$. By Theorem 1 we have $\Delta_{l}(t) \equiv \Delta_{l_{1}}(t)$ $\bmod m(1-t)$. Since $\Delta_{l_{1}}(t)=f(t)$, the proof is complete.

Theorem 4. Let $m$ be a positive integer and $f(t)$ an integral polynomial in $t$. Assume that $f(t)$ satisfies conditions (1) and (2) in the case for a knot and conditions (3) and (4) in the case for a link with the number of components greater than 1. Then there exists an alternating link $l$ with the given number of components such that $\Delta_{l}(t) \equiv f(t) \bmod (1-t) \sigma_{m}\left(t^{2}\right)$.

The proof is quite similar to that of Theorem 3. The only difference here is that instead of the operation described as in Figures 1 and 2, we apply the one described as in Figures 3 and 4, and instead of Theorem 1, we apply Theorem 2. Hence we omit the proof.

\section{REFERENCES}

1. J. W. Alexander, Topological invariants of knots and links, Trans. Amer. Math. Soc. 30 (1928), 275-306.

2. R. H. Crowell, Genus of alternating link types, Ann. of Math. 69 (1959), 258-275.

3. __ Non-alternating link, Illinois J. Math. 3 (1959), 101-120.

4. R. H. Crowell and R. H. Fox, Introduction to knot theory, Ginn, Boston, Mass., 1963.

5. R. H. Fox, Congruence classes of knots, Osaka J. Math. 10 (1958), 37-41.

6. F. Hosokawa, On V-polynomial of links, Osaka J. Math. 10 (1958), 273-282.

7. S. Kinoshita, On Wendt's theorem of knots. II, Osaka J. Math. 10 (1958), 259-261.

8. K. Murasugi, On the Alexander polynomial of the alternating knot, Osaka J. Math. 10 (1958), 181-189. Errata, 11 (1959), 95.

9. 235-248.

10. On alternating knots, Osaka J. Math. 12 (1960), 277-303.

11. R. Riley, A finiteness theorem for alternating links, J. London Math. Soc. (2) 5 (1972), 263-266.

12. H. Seifert, Ueber das Geschlecht von Knoten, Math. Ann. 110 (1934), 571-592.

13. G. Torres, On the Alexander polynomial, Ann. of Math. 57 (1953), 57-89.

Department of Mathematics, Florida State University, Tallahassee, Florida 32306 